\title{
A study using PIV of the Intake Flow in a Diesel Engine Cylinder
}

\author{
Jean Rabault $^{\mathrm{a}, \mathrm{b}}$, Julie A. Vernet ${ }^{\mathrm{a}}$, Björn Lindgren ${ }^{\mathrm{c}}$, P. Henrik Alfredsson ${ }^{\mathrm{a}}$ \\ ${ }^{a}$ Linné FLOW Centre, KTH Mechanics, SE-100 44 Stockholm, Sweden \\ ${ }^{b}$ Dept. Mathematics, University of Oslo, 0316 Oslo, Norway \\ ${ }^{c}$ Scania CV AB, Södertälje, Sweden
}

\begin{abstract}
The admission flow generated by a parallel valve diesel engine cylinder head was investigated by planar and stereoscopic Particle Image Velocimetry in a steady flow test bench through measurements in the swirl and tumble planes. By combining several sets of measurements a full three-dimensional, three-component reconstruction of the flow was made. The flow out of the valves forms a jet which collides with the cylinder wall before flowing down along the wall. Despite the fact that there is no piston a recirculation bubble is formed in the tumble plane. This is due to the entrainment of gas into the jet which needs to be replaced and thereby sets up a counter flow. In the swirl plane complex jet-dominated vortex structures are detected close to the cylinder top. Moving away from the cylinder top, a counter-rotating vortex-pair structure is observed from which a single coherent swirling structure develops further down the cylinder. Some clear differences are observed between the flow at high and moderate valve lifts, which correspond to a distinct change in the swirl intensity. By introducing a strong swirling motion the flow is stabilized which can be seen by tracking the instantaneous position of the swirl centre. For high swirl the variation of the position of the swirl centre decreases substantially.
\end{abstract}

Keywords:

Internal Combustion Engine, Swirling flow, Admission stroke

\section{Introduction}

Four-stroke diesel engines are widely used to power road vehicles, especially heavy duty vehicles, but today also passenger cars. The higher efficiency compared with gasoline engines, together with high power and reliability, explain why they have become the standard engine for heavy duty trucks (Reitz, 2013). Competition between truck manufacturers is therefore driving the research towards higher efficiency as well as higher power, while increasingly strict laws limit the pollutants emissions allowed.

Several strategies can be used to reduce emissions from diesel engines. One consists in so called after treatment of the exhaust gas in order to reduce the soot and NOx content (Kamimoto and Kobayashi, 1991) released into the atmosphere. Such techniques have proved efficient, but also complex and costly to implement. Since the combustion processes are at the heart of the function of the engine, another option consists in reducing the amount of pollutants generated during the combustion, so that the same pollution level can be achieved with less after-treatment in order to reduce the associated costs and complexity.

Optimizing in-cylinder combustion is challenging, since the physics involved is complex and depends on a great number of parameters (see e.g. Lumley, 2001). Fuel admission pressure and diesel-droplet size play a critical role in combustion efficiency (Pozorski et al., 2002) and can also generate flow structures of its own (Zegers et al., 2012; Cao et al., 2000; Roisman et al., 2007). Another element of importance for combustion quality is the flow structures present in the cylinder at the end of the compression stroke when fuel injection takes place (Sun et al., 1996). Flow structures are mostly generated during the admission stroke, and evolve under the influence of volume reduction during the compression stroke. Two general kinds of flow structures are generated during air admission: swirl motion, that corresponds to a coherent rotating motion of the fluid around the cylinder axis, and tumble motion, that corresponds to coherent fluid motion taking place parallel to the cylinder axis (fig. 1). While tumble motion is broken down into turbulence during the compression stroke (Borée et al., 2002), swirl motion survives compression and results in 


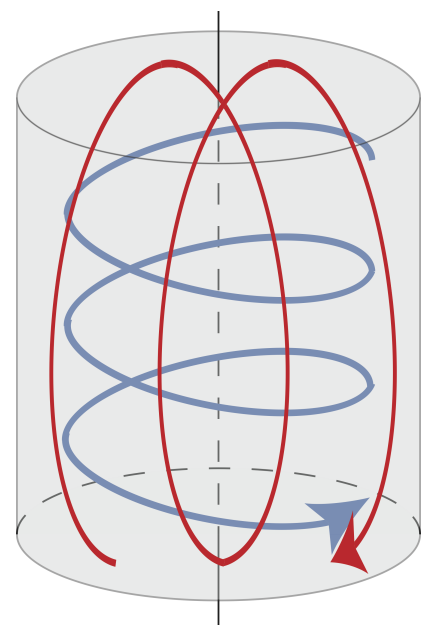

Figure 1: Illustration of Swirl (blue arrow) and Tumble (red arrow) motions.

global flow structures when fuel is injected (Roy and Penven, 1998; Waleffe, 1990). As a consequence, generation of tumble motion is important in spark ignition engines to ensure good propagation of the flame front (Bizon et al., 2010; Peterson and Sick, 2009), while swirl motion is the flow structure that can be used to influence combustion in diesel engines. In diesel engines turbulence is mainly generated by the diesel spray itself. Despite the differences between gasoline and diesel engine design some of the main flow features are similar before combustion. Although engine manufacturers have different design philosophies, one strategy in diesel-engine development is to carefully adapt the level of swirl generated during the admission stroke to obtain the required combustion quality. Performing such fine optimization requires a detailed knowledge and understanding of the underlying flow features.

As a consequence of the importance of the flow during the intake stroke a variety of experiments have been performed on test benches as well as motored piston-cylinder assembly and optical access engines (Stansfield et al., 2007; Dierksheide et al., 2002). Integrated quantities, such as mass-flow rate and swirl intensity, have been investigated for a long time (Crnojevic et al., 1999). Developments of optical measurement techniques have made non-intrusive velocity measurements possible, and techniques such as Laser Doppler Velocimetry (LDV) (Payri et al., 1996; Desantes et al., 1995), Particle Tracking Velocimetry (PTV) (Khalighi, 1991), planar (Cosadia et al., 2007; Reeves et al., 1996), stereoscopic (Vernet, 2012; Rabault, 2015) and tomographic Particle Image Velocimetry (PIV) (Baum et al., 2013, 2014; van Overbrüggen et al., 2013) have been employed for in-cylinder flow studies. However, even using such advanced methods, getting full understanding of the three-dimensional (3D) structure of engine flows is still challenging. One reason for that is the absence of three-dimensional three-component (3D3C) flow information. While planar and stereoscopic PIV give access to the velocity field in only one plane, tomographic PIV remains challenging to perform in a complex geometric configuration representative of an engine and the areas in which information is collected are limited to thin slices. Rotating barrels can be used to change the position where measurement are performed (Brücker, 1997), but such a method is complex to implement and use.

In parallel to experimental investigations, simulations have been used to generate additional knowledge. Starting with Reynolds Averaged Navier-Stokes (RANS) simulations (Basha and Gopal, 2009; Payri et al., 2004), the increase in computer power has allowed more accurate Large Eddy Simulations (LES) (Liu and Haworth, 2010; Hasse et al., 2009; Toledo et al., 2007; Liu et al., 2013), including realistic engine configurations (Söder, 2015; Enaux et al., 2011; Bottone et al., 2012). More recently, Direct Numerical Simulations (DNS) have been performed for idealized engine configurations to investigate the origin of cycle-to-cycle variations (Montorfano et al., 2015; Schmitt et al., 2014). Despite progress accomplished in engine flow simulations (Banaeizadeh et al., 2013), simulating configurations reproducing in detail a true engine remains challenging. As a consequence, experiments cannot be by-passed and are required to build knowledge about specific engine configurations and generate data that can be used to validate simulations (Reitz and Rutland, 1995). 


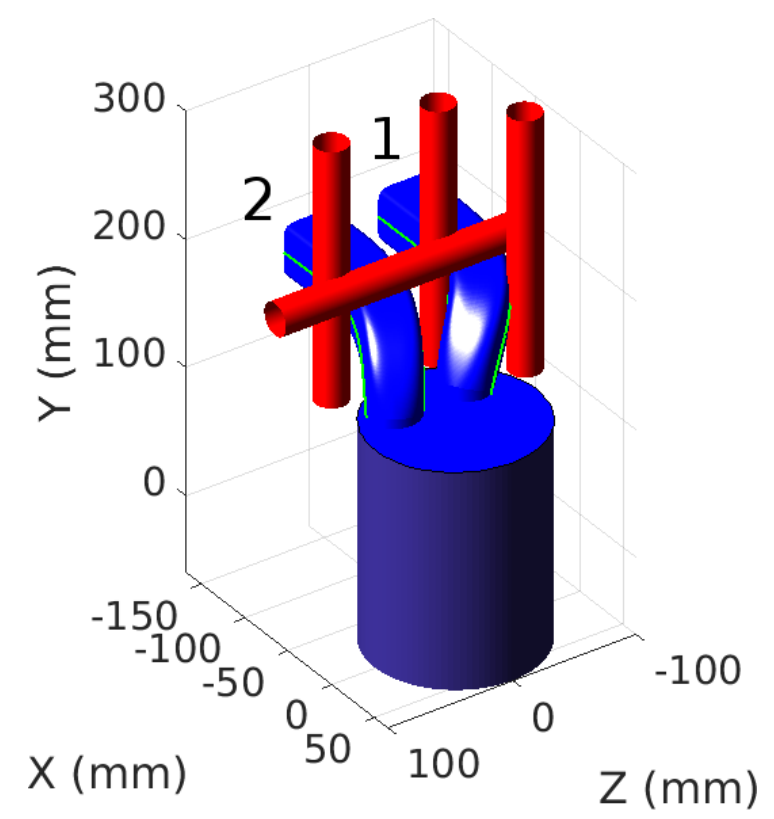

Figure 2: Cylinder head used for performing the measurements. The admission channels and the cylinder are indicated in blue. Red rods indicate limitations in the design space corresponding to screws and cooling channels.

Both experiments and simulations have underlined the importance of admission channel design, port shape, valve positioning (Kang and Reitz, 1999) and cylinder head configuration to achieve a given swirl or tumble intensity (Huang et al., 2008). Other cylinder characteristics, such as the presence and shape of the piston bowl, have proven less important during the admission stroke (Murali Krishna and Mallikarjuna, 2010). While a wide variety of engine configurations have been investigated, some general flow features seem to be shared by most configurations. In particular, the existence of a strong re-circulation bubble in the tumble plane has been noticed by several authors on both diesel and spark ignition engines (Huang et al., 2005; Garth et al., 2007). However, more analysis is needed to understand the associated 3D flow features. Another important flow property is the stability in space and time of the structures obtained (Cosadia et al., 2006; Towers and Towers, 2004; Kapitza et al., 2010; Müller et al., 2010), since more stable structures are expected to give rise to less cycle-to-cycle variations (Enotiadis et al., 1990; Liu et al., 2012; Voisine et al., 2011). A popular technique for trying to assess and understand the origins of such variations is the Proper Orthogonal Decomposition (POD) (Fogleman et al., 2004; Roudnitzky et al., 2006), a method that aims at identifying the most energetic structures in the flow from a set of snapshots (Graftieaux et al., 2001).

In the present paper, both planar and stereoscopic PIV are used to investigate the flow structures generated by a parallel valves diesel-engine cylinder head investigated through test-bench measurements. A complete 3D3C reconstruction of the mean flow field is performed in a large portion of the cylinder, allowing detailed understanding of the flow structures involved. In addition, a parametric study allowed the influence of valve lift on the development of the flow structures in the cylinder to be investigated. Finally, the stability of the swirl structures is investigated.

\section{Experimental configuration}

In this section we describe briefly the cylinder head (sec. 2.1), the test bench facility and the measurement methodology for the integral quantities (sec. 2.2). The PIV system and measurement conditions are described in sec. 2.3 and the methodology to obtain the three-dimensional, three-component (3D3C) reconstruction of the velocity field from the measured stereoscopic data in the tumble planes in sec. 2.4 


\subsection{Cylinder head}

The cylinder head investigated is a prototype used to assess one possible design that could be integrated in the next generation of Scania engines. It features two separate intake ports each equipped with a valve in a parallel configuration and a flat flame face. The cylinder bore is $130 \mathrm{~mm}$, and the engine stroke around $160 \mathrm{~mm}$. The two intake valves are referred to as valve 1 and 2 (see figure 2). Constraints coming from various engineering considerations, such as cooling and material resistance, limit the extent of the design space making it challenging to achieve the desired flow properties in the cylinder. The cylinder head used in the study is a High Swirl Configuration (HSC) prototype. Another configuration is used specifically in section 3.5 for comparison with the HSC and is referred to as the Low Swirl Configuration (LSC). The LSC cylinder head is obtained by changing the geometry of the intake port 1 of the HSC cylinder head so that the cylinder head generates $30 \%$ less swirl at $15 \mathrm{~mm}$ valve lift. For more details, see Rabault (2015).

\subsection{Test bench and measurements of integrated quantities}

All measurements were performed in a steady-flow test bench. A constant pressure difference is applied over the cylinder head, between the entrance of the admission channels and the end of the glass cylinder, and creates the air flow into the cylinder. The valve lift can be adjusted but is kept fixed during each measurement. The corresponding steady configuration is viewed as a simplified version of a diesel-engine cylinder during the intake stroke. The flow during the admission stroke can be viewed as incompressible and both experimental and numerical studies (Vernet, 2012; Söder, 2015) prove that the value of the pressure drop does not influence the flow structures in the cylinder, as long as the Reynolds number is kept high enough. Here a constant pressure drop of $100 \mathrm{~mm} \mathrm{H}_{2} \mathrm{O}$ (981 Pa) was used for all experiments and leads to cylinder mean outflow velocities of 5-10 m/s depending on the valve lift.

In addition to the PIV measurements the test bench is used to measure the two integrated quantities of interest for characterizing the flow. These are the discharge coefficient $\left(C_{D}\right)$ and the Swirl Number $(S N)$. Both quantities are measured in the steady test bench for each valve opening. The discharge coefficient is defined as

$$
\mathrm{C}_{\mathrm{D}}=\frac{q_{\text {meas }}}{q_{\text {ref }}}
$$

with $q_{\text {meas }}$ the actual mass flow rate obtained with the cylinder head and $q_{\text {ref }}$ is reference flow rate related to isentropic flow thorough the valves. The reference flow rate is calculated as

$$
q_{\mathrm{ref}}=\rho A_{0} V_{\mathrm{isen}}
$$

where $\rho$ is the air density and $A_{0}=n \pi d^{2} / 4$ is a reference area related to the valve area $(n$ is the number of valves and $d$ the valve diameter). This area can be chosen more or less arbitrarily, but with this definition one finds that $0<C_{D} \lesssim 1$ which is a suitable range. The flow velocity $V_{\text {isen }}$ corresponds to isentropic expansion for the air regarded as a perfect gas:

$$
V_{\text {isen }}=\sqrt{2 c_{p} T_{1}\left[1-\left(\frac{P_{2}}{P_{1}}\right)^{\frac{\gamma-1}{\gamma}}\right]}
$$

where $c_{p}$ is the specific heat capacity, $\gamma=c_{p} / c_{v}$ the ratio of specific heats, $P_{1}$ and $T_{1}$ the pressure and temperature of the air before admission into the cylinder, and $P_{2}=P_{1}-\Delta P$ is the pressure measured at the exit of the test cylinder, with $\Delta P$ the associated pressure drop. Pressure is assumed constant in the cylinder, so that the pressure drop $\Delta P$ is only due to the cylinder head channels and valves.

The swirl number (SN) is measured by forcing the swirling air into a non-rotational motion using a freely suspended honeycomb placed approximately $230 \mathrm{~mm}$ below the cylinder head. The honeycomb has a length of $45 \mathrm{~mm}$ with hexagonal cells with a diameter of $3 \mathrm{~mm}$ and a wall thickness of $0.1 \mathrm{~mm}$ and the torque exerted on the honeycomb is measured with a load cell. The torque $M_{z}$ is measured together with the mass-flow rate $q_{\text {meas }}$ and $\mathrm{SN}$ can be computed as:

$$
\mathrm{SN}=\frac{2 \rho S M_{z}}{q_{\text {meas }}^{2}}
$$

where the length scale $S$ is the stroke of the piston in the real engine (here $160 \mathrm{~mm}$ ). 


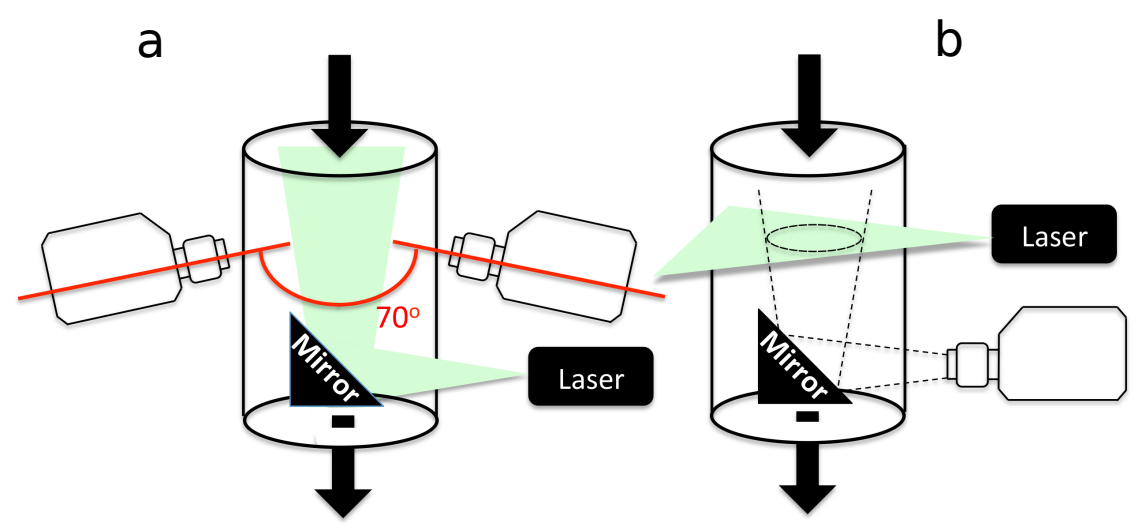

Figure 3: Schematic of the laser and camera arrangement for the tumble plane (a) and swirl plane (b) PIV measurements.

\begin{tabular}{||cccc||}
\hline Configuration & Measurement type & Valve lift & Top field of view to cylinder top \\
\hline \hline HSC & S-PIV, 18 Tumble planes & $15 \mathrm{~mm}$ & $2 \mathrm{~cm}$ \\
\hline HSC & S-PIV, 18 Tumble planes & $15 \mathrm{~mm}$ & $12 \mathrm{~cm}$ \\
\hline HSC & Mono PIV, Swirl plane & 3 to $15 \mathrm{~mm}, 3 \mathrm{~mm}$ stepping & $2,4,6,8,10,12,14,23,40 \mathrm{~cm}$ \\
\hline LSC & Mono PIV, Swirl plane & $15 \mathrm{~mm}$ & $40 \mathrm{~cm}$ \\
\hline
\end{tabular}

Table 1: Summary of all PIV measurements performed.

\subsection{PIV system}

The cylinder used in the test bench is made of industry-grade glass and makes the flow inside the cylinder optically accessible. The PIV measurements were performed in both the swirl and tumble planes. A Nd:YAG laser producing a beam of wavelength $532 \mathrm{~nm}$ was used together with $4 \mathrm{Mpx}, 14$ bit dynamic range CCD cameras, at a frame rate of $7 \mathrm{~Hz}$. Seeding particles of typical size 1-5 $\mu \mathrm{m}$ were obtained from a smoke generator burning glycol-like oil. Stereoscopic PIV (S-PIV) was used in the tumble planes but due to the difficulty of getting good optical access and suppressing laser reflections, only planar PIV measurements were performed in the swirl planes. Tumble plane measurements were performed with the two cameras looking through the glass cylinder, with the laser shot from under the cylinder head using an inclined (45 degrees) mirror (figure 3(a)). Scheimpflug adaptors were used on both cameras and the Davis LaVision 8.0 software and calibration plate were used to perform stereoscopic calibration and to ensure that the whole field of view was in focus for both cameras. The angle between the axis of the two cameras is ideally 90 degrees, but due to space constraints on the test bench the angle used was about 70 degrees. When performing swirl plane measurements, the laser was shot through the glass cylinder perpendicular to its axis, and the camera captured the flow by looking through the mirror (figure 3(b)). All PIV measurements performed are summarized in Table 1.

An uncertainty analysis based on results from one plane of S-PIV data was first made to determine the number of independent snapshots required to reach an accuracy in the mean flow better than $5 \%$. For performing the analysis we assumed that the velocity at one point in space, regarded as a stochastic process $X_{i}$, follows a Gaussian distribution. The Gaussian distribution of the velocity at one point is the consequence of both turbulence and the uncertainty inherent to the PIV algorithm. This leads to the equation (Bendat and Piersol, 2000):

$$
\operatorname{Pr}\left(\bar{x}-z_{\alpha} \frac{\sigma_{x}}{\sqrt{N}}<\mu_{x}<\bar{x}-z_{\alpha} \frac{\sigma_{x}}{\sqrt{N}}\right)=\alpha,
$$

where $\operatorname{Pr}$ is the probability that the mathematical assertion is true, $N$ is the total number of snapshots used to compute the mean field, $z_{\alpha}$ is a tabulated quantity that depends on the probability $\alpha, \mu_{x}$ is the real mean of the velocity at the point considered, $\bar{x}$ and $\sigma_{x}$ are respectively the non biaised mean estimator and standard deviation. Here, we take $\alpha=0.95$. The non biaised mean estimator is computed as: 

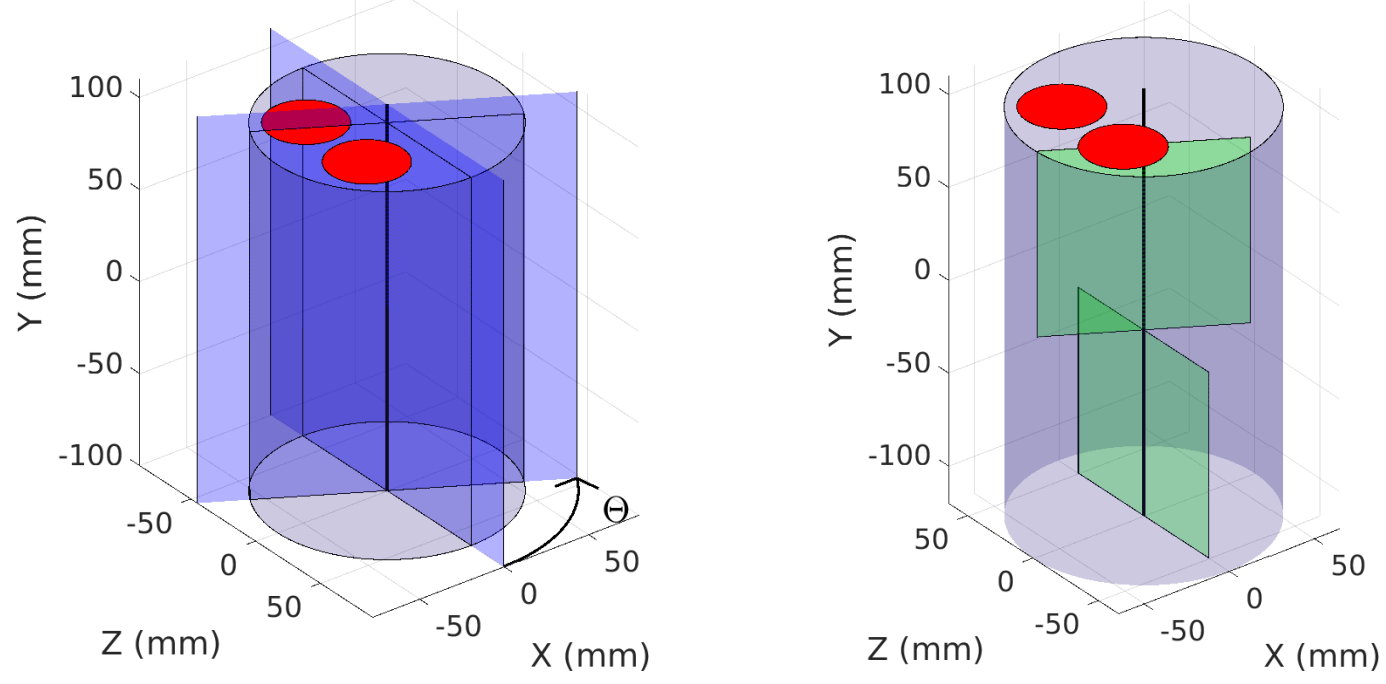

Figure 4: Left: illustration of the method used for 3D3C reconstruction. The cylinder head is rotated while the cylinder and the PIV apparatus stay fixed, which allows to perform measurements without the need to calibration each time a new plane is investigated. The reference plane is the one perpendicular to $\mathrm{X}$ axis. The position of a tumble plane is described through its angle relatively to the reference plane. Right: field of view corresponding to the velocity fields presented in figure 6 .

$$
\bar{x}=\frac{1}{N} \sum_{i=1}^{N} X_{i}
$$

Since the standard deviation $\sigma_{x}$ of the process considered is unknown, it is estimated by the non biased standard deviation estimator $s_{x}$, which is defined as:

$$
s_{x}^{2}=\frac{1}{N-1} \sum_{i=1}^{N}\left(X_{i}-\bar{x}\right)^{2}
$$

Using this framework of analysis, a minimum of 300 snapshots were found to be necessary for computing mean velocity fields satisfying the $5 \%$ accuracy target in the active part of the flow. More details about the points used for performing the analysis and the verification of the Gaussian approximation can be found in Rabault (2015).

\subsection{D3C methodology}

S-PIV gives all three velocity components in the measurement plane but it remains difficult to fully comprehend the three-dimensional (3D) flow from two-dimensional planes. Instead a full 3D3C reconstruction in the whole domain was made by using a set of 18 S-PIV tumble plane measurements (Rabault, 2015). The measurements were performed by rotating the cylinder head while keeping the same S-PIV camera configuration, as indicated in figure 4 . The angle between two consecutive measurement planes is 10 degrees and since the measurement planes extend to both positive and negative local $\mathrm{X}$ values, this covers a whole cylindrical measurement area. The 3D3C reconstruction is then made from the different fields through a Matlab script. With such a method, the 3D velocity field in a plane is observed from different relative angles without moving the cameras. This allows all the measurements for a 3D reconstruction to be made without changing the PIV setup and without the necessity to go through time-consuming optics tuning and calibration. However it has limitations as compared to tomographic PIV, since snapshots are only available in each measurement plane and for the whole domain reconstruction has to be done after the mean flow is available. Nevertheless the extent of the domain in which 3D3C velocity field is available is much larger than what was presented in Tomo-PIV investigations, in which the measurement domain was limited to a thin, small rectangular volume (Baum et al., 2014). 

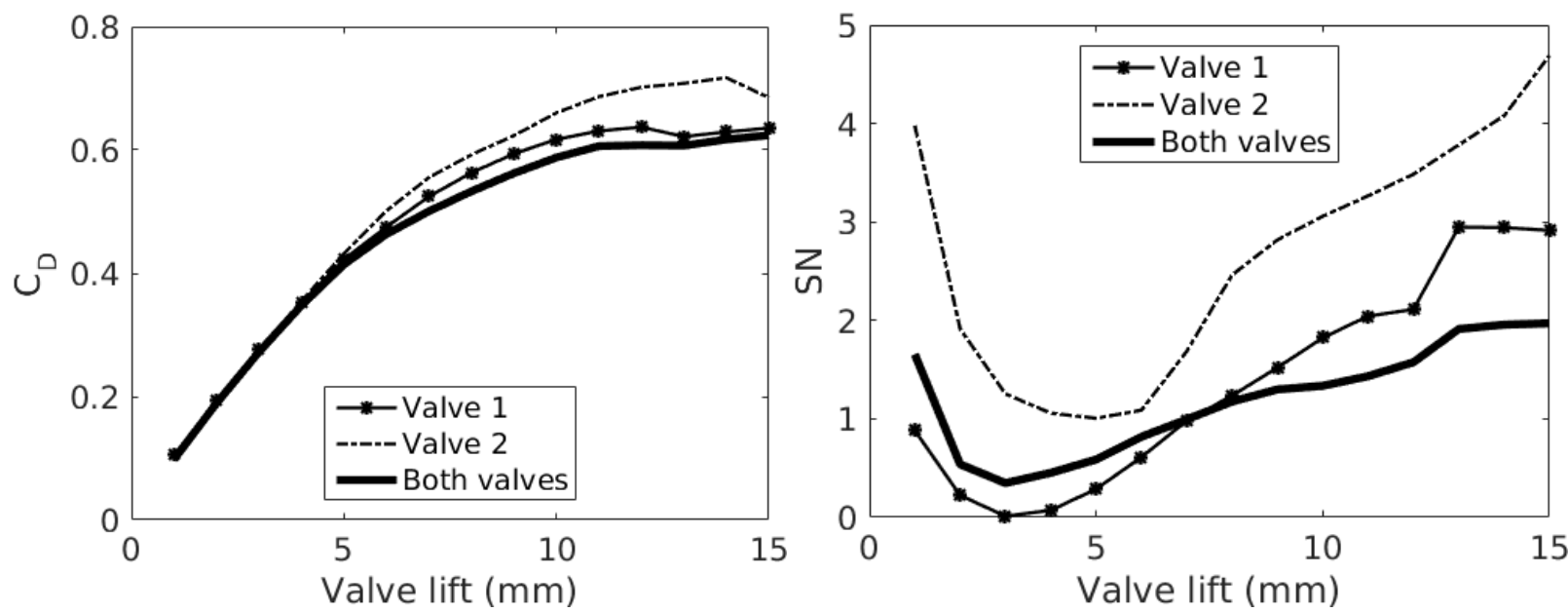

Figure 5: Values of the $C_{\mathrm{D}}$ and $\mathrm{SN}$ coefficients depending on valve lift for the cylinder head investigated. The performance of each valve taken separately is also presented.

\section{In-cylinder measurement results}

\subsection{Measurement of integrated quantities}

Both the swirl number $(\mathrm{SN})$ and discharge coefficient $\left(\mathrm{C}_{\mathrm{D}}\right)$ were measured for valve lifts ranging from 1 to $15 \mathrm{~mm}$. This range of valve lifts corresponds to the values encountered in real engines. The data obtained for each valve separately and the two valves together are presented in figure 5 and as seen the results depend non-linearly on the valve lift. The values with both valves open are also quite different from the addition of the two valves taken separately, which means that the flow obtained from the two valves strongly interact. Valve 1 tends to generate low swirl, which is typical of parallel-valve configuration. A jump in the swirl number is observed around a valve lift of $12 \mathrm{~mm}$ for valve 1 and both valves together.

\subsection{PIV measurements in the tumble plane}

Stereoscopic PIV measurements in tumble planes were performed both close to the cylinder top and lower down in the cylinder for a $15 \mathrm{~mm}$ valve lift, as presented in figure 6 . The corresponding field of view positions are visible in figure 4. Taking away laser reflections in this configuration was challenging, and in addition particles tended to stick on the interior of the glass cylinder creating an oily layer that blurred the images as time went on. As a consequence, while the glass cylinder was cleaned after each run and masks were used to diminish the impact of reflections on image quality, the velocity fields obtained have some level of noise. However this has no consequence on the possibility to gain physical understanding of the flow from the velocity fields obtained. A clear jet structure is observed close to the cylinder top from the in-plane velocity component, and a strong recirculation bubble is observed both close to the cylinder top and lower down in the cylinder. The out-of-plane velocity component, that corresponds to swirl motion, is influenced by the jets close to the valves while clear swirling motion develops further away from the cylinder top.

Using the data from S-PIV measurements, 3D3C reconstructions were performed for a $15 \mathrm{~mm}$ valve lift in the two different positions in the cylinder, as presented in figure 7. The arrows indicate the velocity in the swirl plane and the colour scheme shows the vertical velocity. The jet structure is visible near the valves and the downward motion of the fluid is localized near the wall opposite to the valves, in the region where the jets impact on the cylinder wall. A small counter-rotating jet, coming from valve 2 , is also observed in the figure. To obtain strong swirl this counter-rotating jet is unwanted, however it originates from the difficulty to introduce the flow tangentially to the cylinder wall in a parallel-valve configuration. Further down in the cylinder a clear coherent swirling motion is observed. The vertical velocity confirms the existence of a strong recirculation bubble, in agreement with previous results, both experimental and numerical, in various engine configurations (Khalighi, 1991; Payri et al., 2004). However it is the first time as far as the authors are aware that the full 3D picture can be obtained from an experiment in a wide volume of a complex 

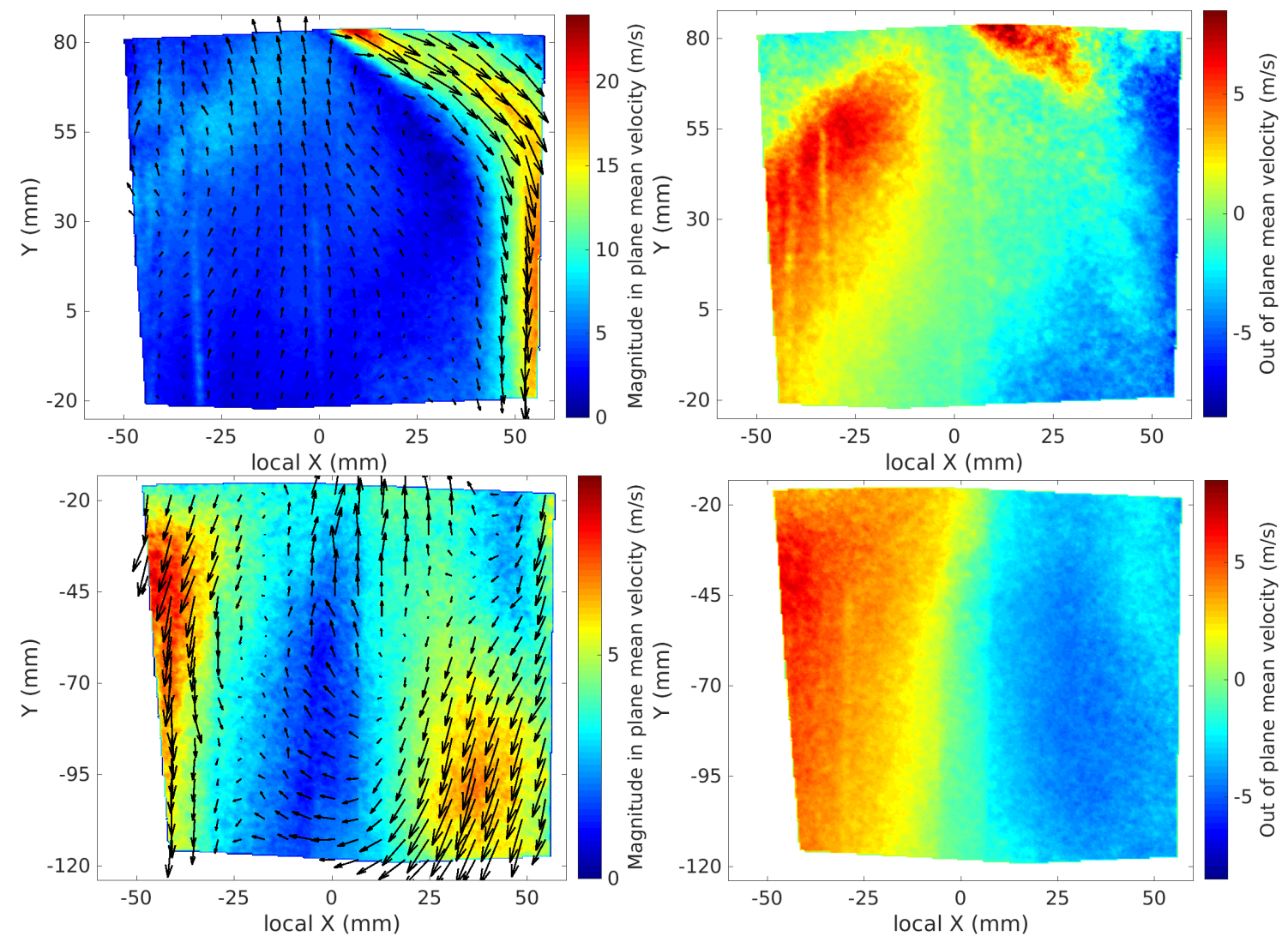

Figure 6: Results of PIV measurements at $15 \mathrm{~mm}$ valve lift in tumble planes close to the cylinder top (top, relative angle to the reference plane 60 degrees) and lower down in the cylinder (bottom, relative angle to the reference plane 0 degrees). Left: in-plane velocity magnitude and direction. Right: out-of-plane velocity component. The two measurement planes correspond to those indicated in figure 4. 


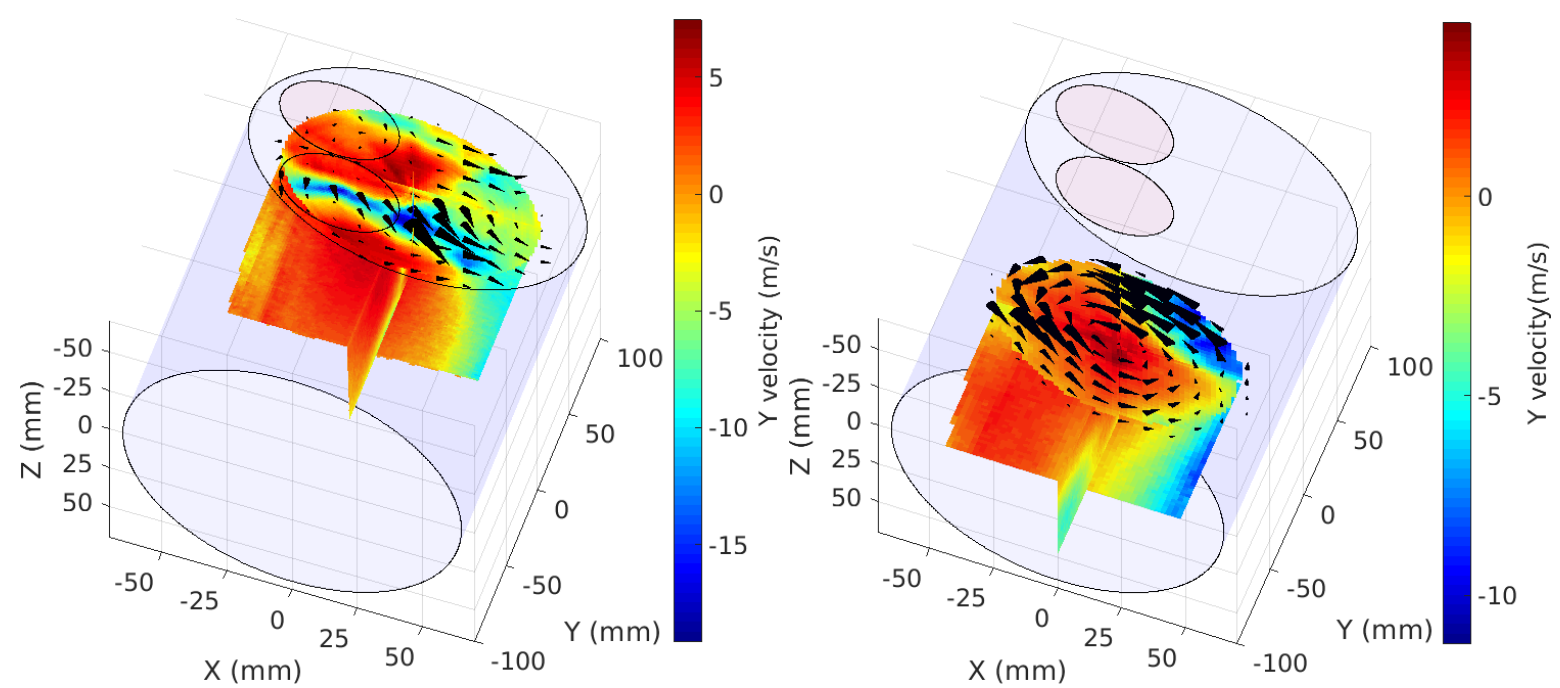

Figure 7: 3D3C reconstruction of the flow at both the high (left, the swirl plane plotted corresponds to a distance to the cylinder top of $2 \mathrm{~cm}$ ) and low (right, the swirl plane plotted corresponds to a distance to the cylinder top of $12 \mathrm{~cm}$ ) measurement positions for a $15 \mathrm{~mm}$ valve lift.

experimental setting representing a real engine cylinder head, previous 3D measurements being limited to a small portion of the cylinder (Baum et al., 2013; van Overbrüggen et al., 2013).

\subsection{PIV measurements in the swirl plane}

By making a 3D3C reconstruction a better general understanding about the flow is obtained, but the time required to carry out the measurements necessary to obtain one 3D picture makes it difficult to perform parametric studies of the influence of e.g. valve lift on flow features. The reconstruction process also implies that the accuracy of the information available in each swirl plane is limited. Finally, laser reflections on the glass cylinder wall make it difficult to observe the flow field close to the walls. As a complement, detailed influence of both the valve lift and the distance to the cylinder top on the structures present in the swirl plane was studied from planar PIV measurements.

The distance between the swirl plane in which measurements were performed and the cylinder top ranged from $2 \mathrm{~cm}$ to $40 \mathrm{~cm}$. It was not possible to perform measurements closer than $2 \mathrm{~cm}$ away from the cylinder top due to laser light reflections on the valves. To investigate flow stability, measurements were performed as far as $40 \mathrm{~cm}$ from the cylinder top, which is more than the real engine stroke. Indeed, based on the mean vertical flow velocity, the air takes as much time to go from the valves to a distance of $40 \mathrm{~cm}$ down in the cylinder as the period of one admission and compression stroke. Therefore, even if we cannot investigate some of the important flow properties that take place during the compression stroke, we expect to get an indication of the general trend in flow stability and swirling patterns that are obtained when the fuel injection takes place. As presented in figure 8 (top), for moderate valve lift (less than $12 \mathrm{~mm}$ ), the flow pattern in the swirl plane evolves from a complex situation where the jets are active near the valves, to a counter-rotating double vortex structure further away from the cylinder top, from which the strongest vortex becomes predominant and a single coherent swirling structure can be observed further down in the cylinder. For larger valve lifts ( $12 \mathrm{~mm}$ and above), the same general trend is observed but the counter-rotating double vortex is less developed and gets perturbed by additional vortices (figure 8 , middle). Those observations are in good agreement with previous investigations of both diesel of spark ignition cylinder heads, in which similar double vortex structures have been observed (Vernet, 2012; Huang et al., 2005). The larger the valve lift, the closer to the cylinder top the double vortex structure and the single vortex structure can be observed (figure 8, bottom). While a double vortex structure can still be observed at moderate valve lifts $12 \mathrm{~cm}$ from the cylinder top, a clean swirling motion is observed for the maximum valve lift. The valve lift over which the double vortex becomes less visible corresponds to the jump in the swirl number of valve 1 that can be seen in figure 5 .

Streamlines are useful for analyzing flow patterns, but they do not contain information about which parts of the flow that are the most active. Therefore additional information can be obtained by looking at both in-plane velocity 

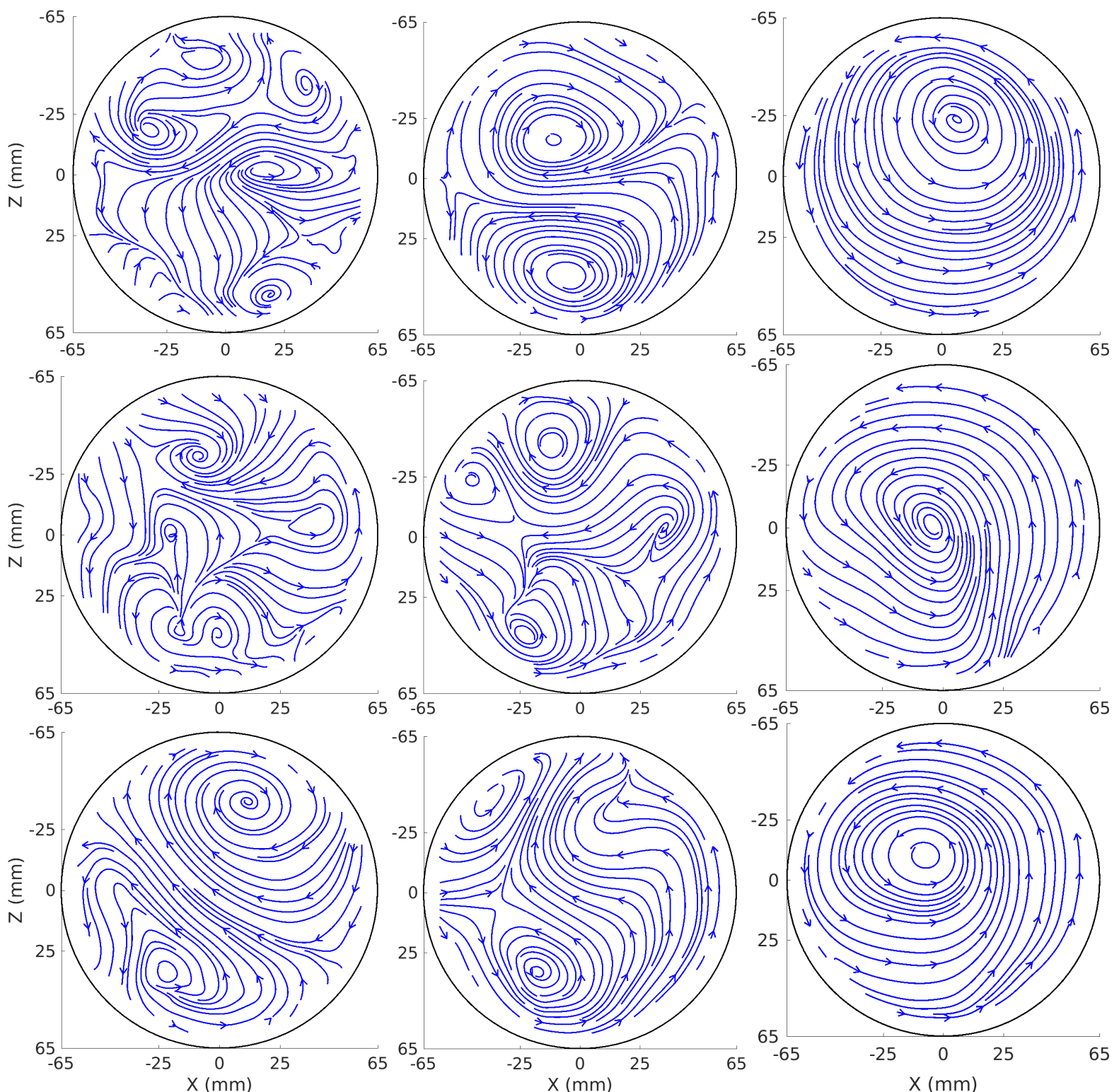

Figure 8: Evolution of the flow structures in the swirl plane with valve lift and distance to the cylinder top. Top: valve lift $6 \mathrm{~mm}$, distance to the cylinder top 2, 10, $40 \mathrm{~cm}$ (from left to right). Middle: valve lift $12 \mathrm{~mm}$, distance to the cylinder top $4,8,14 \mathrm{~cm}$ (from left to right). Bottom: distance to the cylinder top $12 \mathrm{~cm}$, valve lift 3, 9, $15 \mathrm{~mm}$ (from left to right). 

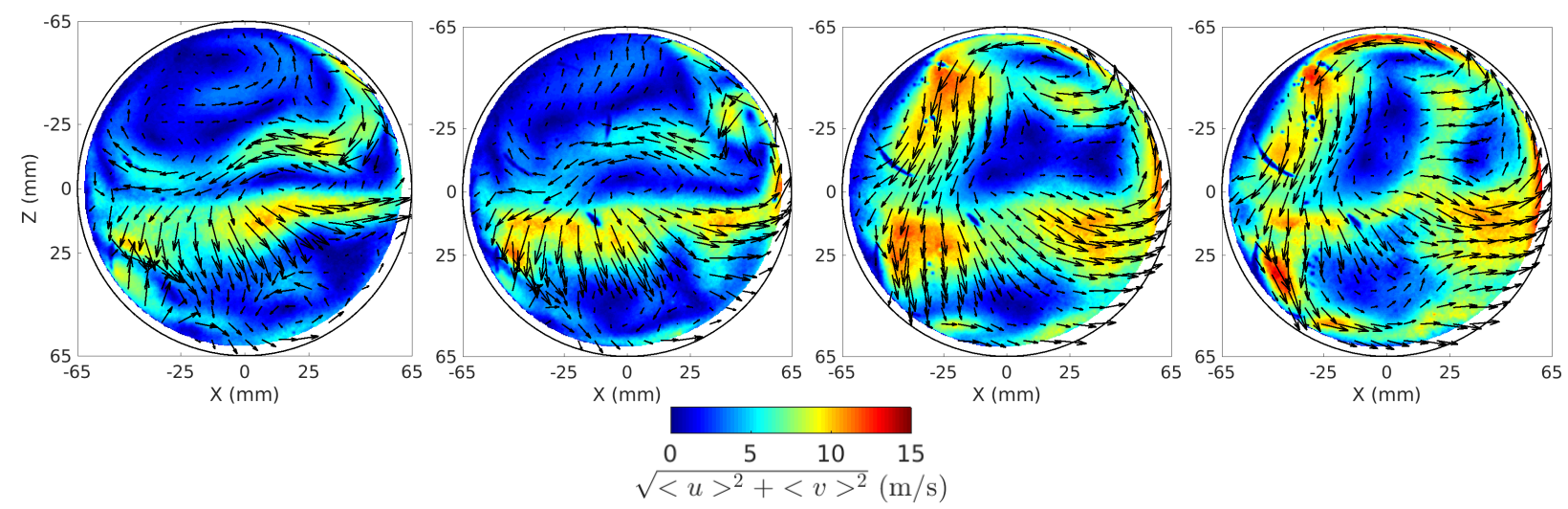

$$
\begin{array}{cccc}
0 & 5 & 10 & 15 \\
\sqrt{\left.\langle u\rangle^{2}+<v\right\rangle^{2}} & (\mathrm{~m} / \mathrm{s})
\end{array}
$$

Figure 9: In plane velocity magnitude (colour) and direction (arrows), $2 \mathrm{~cm}$ from the cylinder top, for valve lifts of 6, 9, 12 and $15 \mathrm{~mm}$ (left to right). A transition in the flow pattern, corresponding to the jump in swirl coefficient presented in figure 5, can be observed between valve opening 9 and $12 \mathrm{~mm}$. Reflections on the valves are visible on the left part of the figures. The colorbar applies to all figures.

magnitude and velocity direction. Figure 9 presents the flow developing $2 \mathrm{~cm}$ from the cylinder top for valve lifts under (top) and over (bottom) $12 \mathrm{~mm}$, around which a jump in the swirl coefficient was observed in figure 5 . Due to the very short distance to the cylinder top, reflections make the valves visible in the figures seen as distortions in the computed velocity field under the edges of the valves. A clear transition between small and large valve lifts in the flow is observed. While the flow developing under valve 2 remains similar in both situations, the flow under valve 1 goes through a transition when the valve lift is increased. At low valve lifts the active regions generated by valve 1 are mostly going against the direction of the swirl motion observed further from the cylinder top. By contrast, for high valve lift, the active flow regions under valve 1 mostly follow the direction of the general swirling motion. This transition was difficult to notice from observation of the streamlines.

The development of the flow with increasing distance to the cylinder top, for 6 and $12 \mathrm{~mm}$ valve lifts, is presented in figures 10 and 11. As was noticed in figure 8, the single swirling vortex is observed closer to the cylinder top at higher valve lift. The active flow regions at distances to the cylinder top before a single swirling structure is observed are different between 6 and $12 \mathrm{~mm}$ valve lift. While at low valve lift the active flow region is localized mainly in the cylinder centre region and do not contribute to the flow momentum around the cylinder axis, at higher valve lift high in-plane velocities are observed near the cylinder wall and the motion orientation is the same as in the swirling structure observed further from the cylinder top.

\subsection{Calculation of swirl number and discharge coefficient from planar PIV data close to the cylinder top}

The momentum generating the swirl motion in the cylinder is driven by the jets coming out of the valves. As seen in figure 6, those jets are injected at an angle $\alpha$ around 37 degrees and can be separated from the rest of the flow, since they correspond to high velocity regions. One can therefore compute the injected mass flow rate $q_{i n j}$ and momentum $\mathbf{M}_{\text {inj }}$, by setting a threshold velocity $V_{\text {thres }}$ for the in-plane velocity obtained from the planar PIV measurements over which the flow is considered to belong to a jet, injected in the cylinder at the angle $\alpha$. The expression for the mass flow rate and momentum injected by the jets are then:

$$
\begin{gathered}
q_{\text {inj }}=\sum_{l} \rho\left|\mathbf{u}_{\mathbf{l}}\right| \tan (\alpha) \Delta A_{l} \\
\mathbf{M}_{\text {inj }}=\sum_{l} \rho\left(\mathbf{r}_{\mathbf{l}} \times \mathbf{u}_{\mathbf{l}}\right)\left|\mathbf{u}_{\mathbf{l}}\right| \tan (\alpha) \Delta A_{k}
\end{gathered}
$$

where $l$ is the list of indices for which in plane velocity modulus is above $V_{\text {thres }}, \mathbf{r}_{1}$ the vector from swirl plane centre to the point considered and $\mathbf{u}_{\mathbf{l}}$ the associated local velocity. A reference value of $V_{\text {thres }}=3 \mathrm{~m} / \mathrm{s}$ was used for valve lifts $3,6,9 \mathrm{~mm}$ while a reference value of $V_{\text {thres }}=6 \mathrm{~m} / \mathrm{s}$ was used for valve lifts 12 and $15 \mathrm{~mm}$, for which stronger flows are measured. The swirl number is then computed as: 

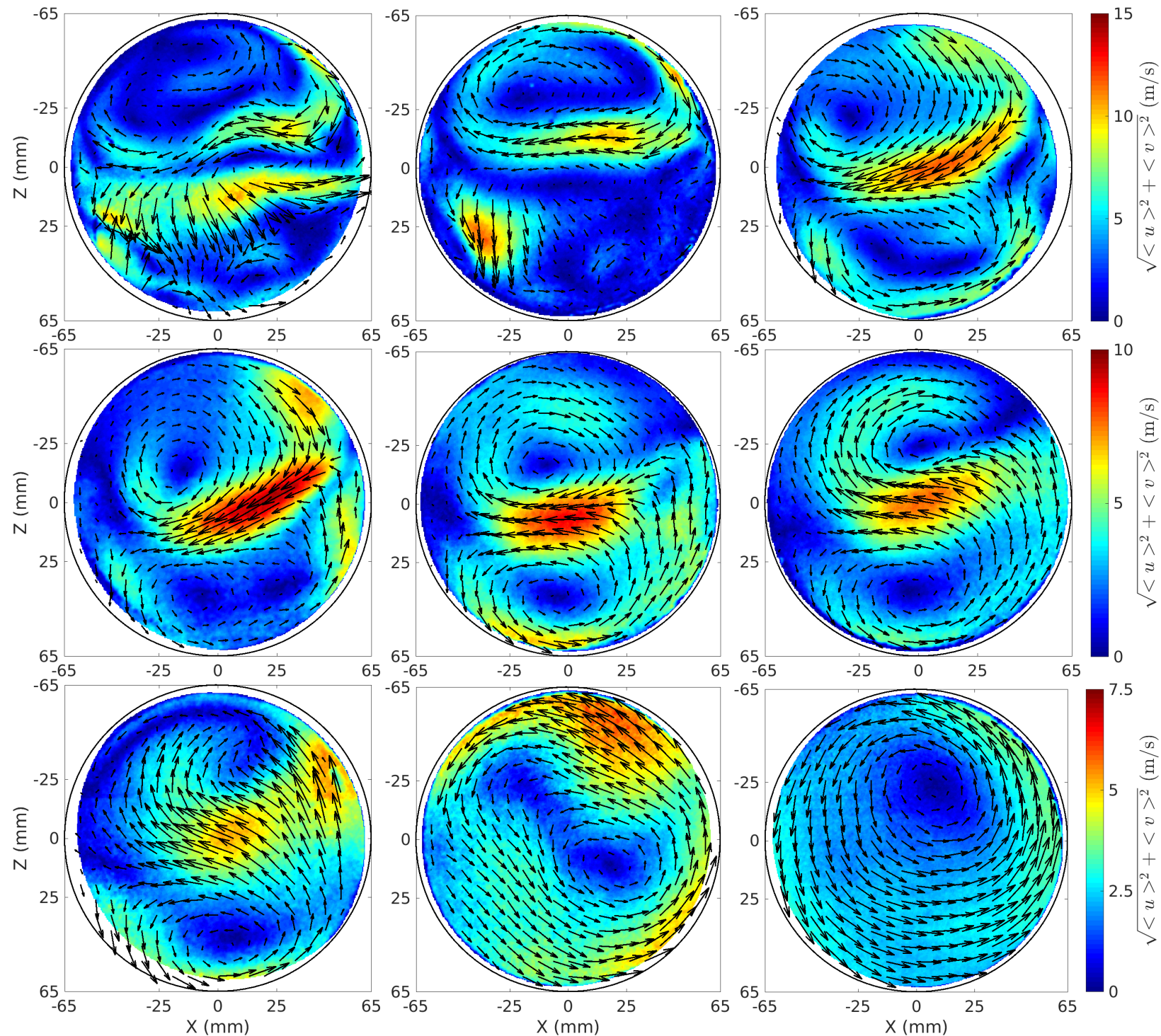

Figure 10: Evolution of the in plane velocity magnitude (colour) and direction (arrows) at a $6 \mathrm{~mm}$ valve lift with increasing distance to the cylinder top (left to right, top to bottom, distance 2, 4, 6, 8, 10, 12, 14, 23, $40 \mathrm{~cm}$ ). The colorbars apply to each figure in a line. 

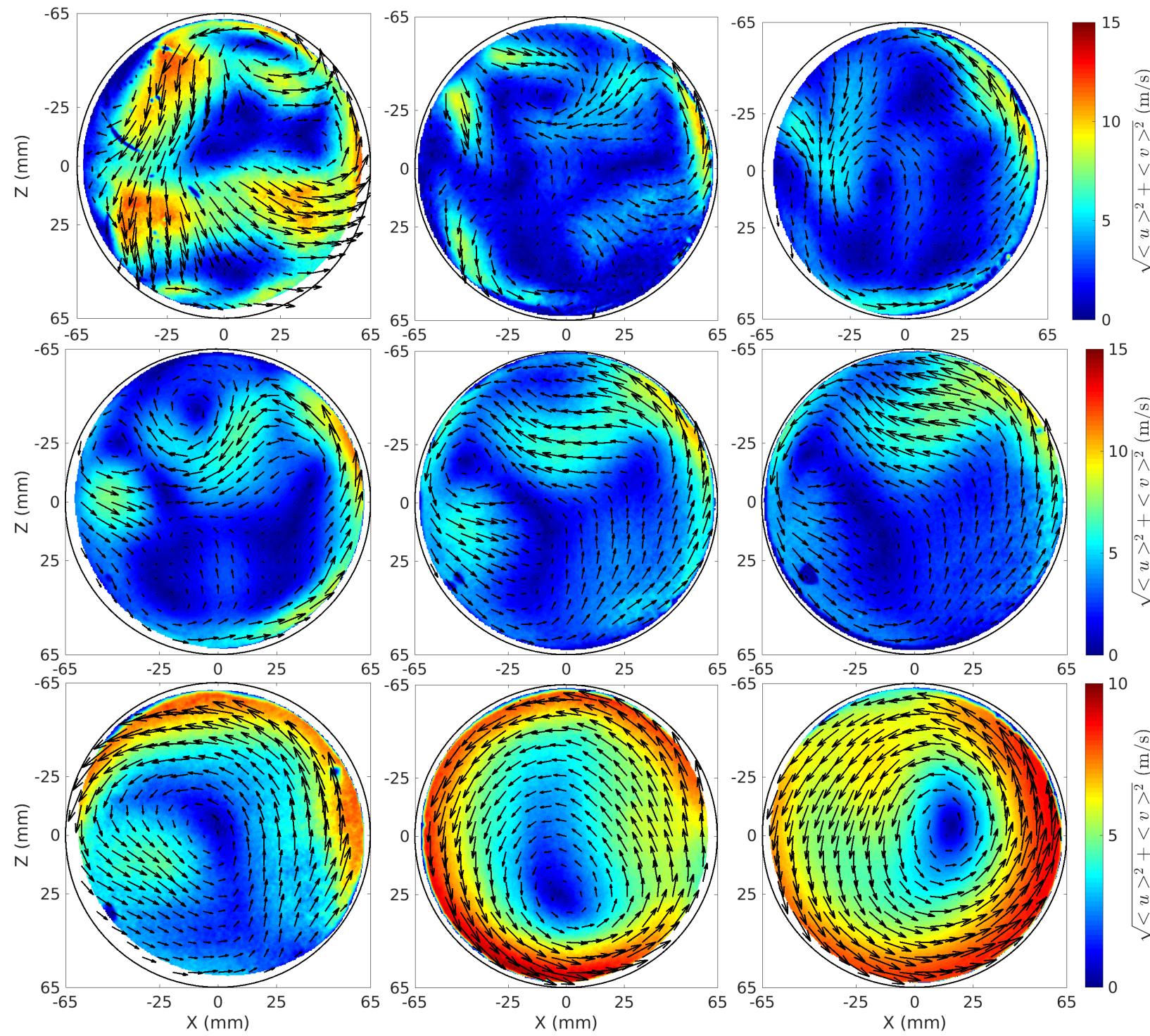

Figure 11: Evolution of the in plane velocity magnitude (colour) and direction (arrows) at a $12 \mathrm{~mm}$ valve lift with increasing distance to the cylinder top (left to right, top to bottom, distance 2, 4, 6, 8, 10,12,14,23, $40 \mathrm{~cm}$ ). The colorbars apply to each figure in a line. 

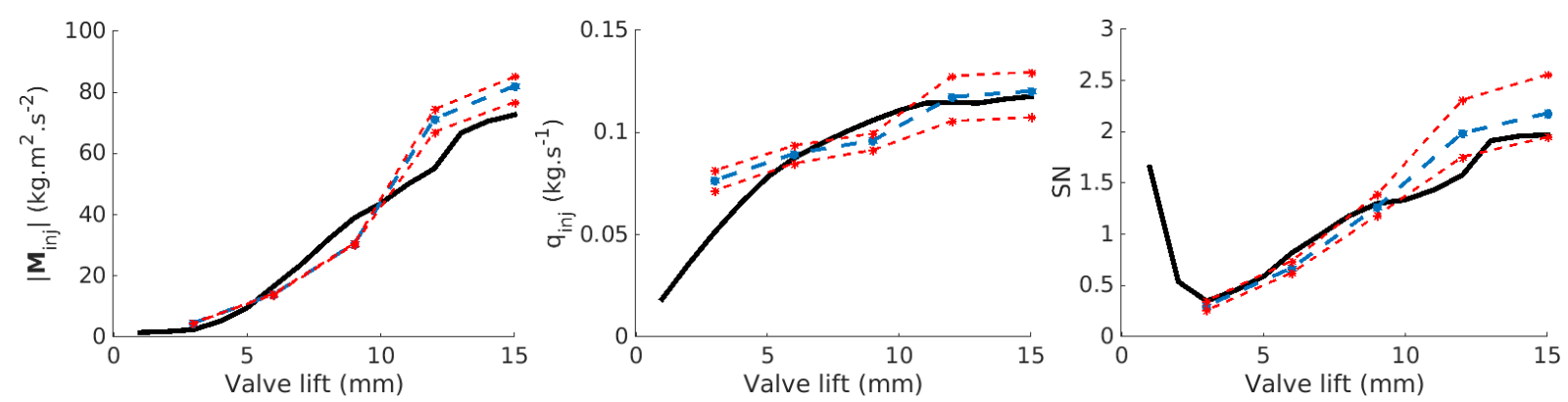

Figure 12: Moment, mass flow rate and SN value obtained from the paddle measurements (black) and the planar PIV data $2 \mathrm{~cm}$ from the cylinder top (blue). The curves obtained by increasing and decreasing the value of $V_{\text {thres }}$ by $10 \%$ are presented in red.

$$
\mathrm{SN}=\frac{2 \rho S\left|\mathbf{M}_{\text {inj }}\right|}{q_{\text {inj }}^{2}}
$$

where $S=160 \mathrm{~mm}$ is the engine stroke. Results obtained with this approximation, $2 \mathrm{~cm}$ from the cylinder top, correspond to the blue curves presented in figure 12. While the paddle measurements are fast to perform, and data is therefore available for valve lifts 1 to $15 \mathrm{~mm}$ with $1 \mathrm{~mm}$ steps, PIV measurements were only performed for valve lifts of 3 to $15 \mathrm{~mm}$, with $3 \mathrm{~mm}$ steps. The red curves correspond to threshold velocities $V_{\text {thres }} 10 \%$ higher and lower than the reference value at the corresponding valve lift. By changing the value of $V_{\text {thres }}$ a larger spread in the results is observed at high valve lift than low valve lift, but the general trends are well captured in all cases.

\subsection{Analysis of swirling structure stability}

Far downstream the cylinder top, a well defined mean swirling structure is observed. The fluctuations in time of the corresponding structure are directly related to flow variations, and a more stable structure is therefore expected to be beneficial for reducing cycle-to-cycle variations in an engine. To study the stability of the swirling structure, its rotation centre is tracked in each instantaneous snapshot used to generate the mean velocity field.

Sophisticated generic algorithms have been described in a variety of previous works for performing vortex core identification (Berson et al., 2009; Kida and Miura, 1998; Finn and Boghosian, 2006). However the relative simplicity of the present situation, implied by the limitation to $2 \mathrm{D}$ velocity fields and the existence of a well defined mean structure, allows the use of simpler methods (Rabault, 2015). Here a two step algorithm is used for performing the tracking. Regions of low velocity magnitude $c$ are first identified on the planar PIV snapshot by minimizing the function:

$$
C_{1}(x, z)=4 c(x, z)+c(x+\mathrm{d} x, z)+c(x-\mathrm{d} x, z)+c(x, z+\mathrm{d} z)+c(x, z-\mathrm{d} z)
$$

where $\mathrm{d} x$ and $\mathrm{d} z$ are taken equal to the resolution of the PIV measurement, so that the local fluctuations of the PIV snapshot are reduced. A second step is then performed in the area where the minimum of $C_{1}$ was identified to find a more exact position. During this step, the minimum of the function $C_{2}$ is selected, where:

$$
C_{2}(x, z)=\sum_{k=1}^{N}\left[r_{x, k} u_{z}\left(P_{k}\right)-r_{z, k} u_{x}\left(P_{k}\right)\right]
$$

with $\left(r_{x, k}, r_{z, k}\right)=\overrightarrow{M P}_{k}$, where $P_{k}$ is a list of $N$ points equally spaced on the circle of centre $M=(x, z)$ and radius $R$, and $R$ is taken equal to $8 \mathrm{~mm}$. The function $C_{2}(x, z)$ is therefore proportional to the flow circulation around the point $M$, computed using a discrete integration on a circle of radius $8 \mathrm{~mm}$.

The final step is then to compute the Probability Density Function (PDF), defined as:

$$
\operatorname{PDF}(x, z)=\frac{\operatorname{Pr}[(x-\mathrm{d} x / 2, x+\mathrm{d} x / 2),(z-\mathrm{d} z / 2, z+\mathrm{d} z / 2)]}{\mathrm{d} x \mathrm{~d} z}
$$



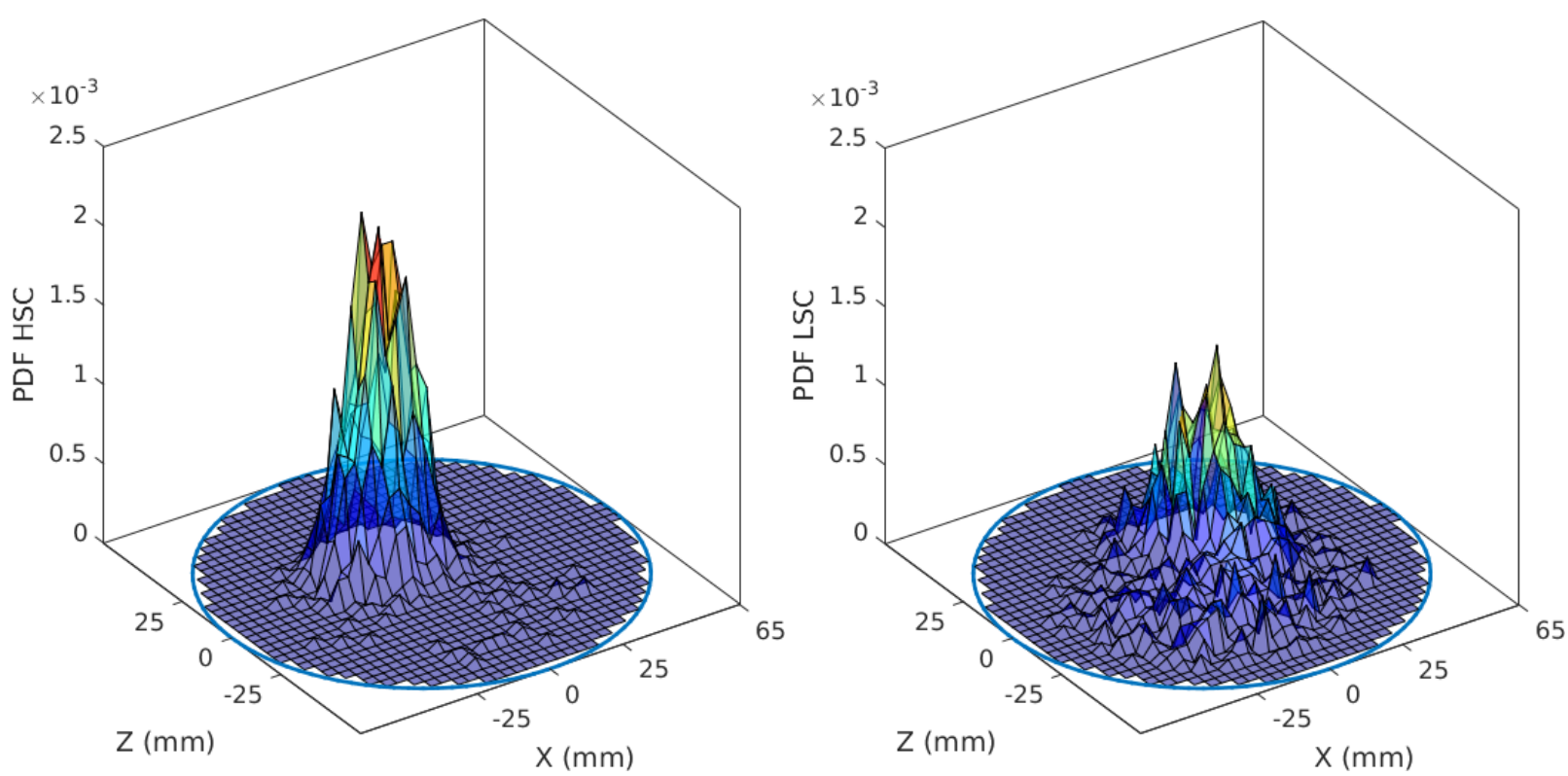

Figure 13: Comparison of the Probability Density Functions for the swirl centre position $400 \mathrm{~mm}$ from the cylinder top at $15 \mathrm{~mm}$ valve lift, corresponding to the cylinder head studied (left, HSC), and a lower swirl number version of the same cylinder head (right, LSC).

where $\operatorname{Pr}[(x-\mathrm{d} x / 2, x+\mathrm{d} x / 2),(z-\mathrm{d} z / 2, z+\mathrm{d} z / 2)]$ is the probability that the instantaneous swirl centre lies in the element of centre $(x, z)$ and side sizes $\mathrm{d} x$ and $\mathrm{d} z$ respectively. The corresponding probability is estimated from the empirical distribution obtained for the list of swirl centre positions.

Such an analysis is performed using 1500 snapshots for the two versions (High Swirl Configuration HSC and Low Swirl Configuration LSC) of the cylinder head, $40 \mathrm{~cm}$ from the cylinder top at $15 \mathrm{~mm}$ valve lift. The probability density distributions of the swirl centre obtained for each cylinder head are presented in figure 13. In the HSC case the swirl centre is distributed within a region of rather limited extent, whereas the swirl centre distribution for the LSC head covers a much wider area of the swirl plane and is less regular. This shows that the LSC investigated not only generates lower values of the swirl number compared to the HSC, but that the flow obtained is also less stable in time. This may translate into larger cycle-to-cycle variations for the engine, known to reduce performance.

\section{Conclusions}

The flow generated by a prototype of a diesel engine cylinder head has been investigated by means of planar and stereoscopic PIV in a steady flow-test bench. A complete 3D3C reconstruction of the mean flow in a major part of the cylinder was obtained for the first time to the authors knowledge, by combining measurements taken in 18 different tumble planes. Such a reconstruction allows the complex 3D structures developing during the admission stroke to be seen. The flow is dominated by a strong jet structure close to the valves. Those jets collide with the cylinder wall opposite to the intake ports before continuing down in the cylinder. A strong recirculation bubble is detected, in good agreement with previous studies. The flow structures then evolve towards more ordered large scale patterns with increasing distance from the cylinder top. At an intermediate distance from the cylinder top, a double vortex structure is detected which ultimately breaks down and evolves into a single swirling structure far from the cylinder top.

Valve lift influences the flow structures in two ways. The transition to more ordered flow structures occurs closer to the cylinder top at higher valve lifts. The strength and regularity of the associated flow structures, including the swirling structure obtained far from the cylinder top, are increased with higher valve lifts. Those changes can be detected in the structure of the jets coming out of the valves. In particular a transition is found for a valve lift around $12 \mathrm{~mm}$, above which valve 1 generates much higher swirl than for lower valve lifts. All those observations of flow features obtained using PIV are in close agreement with results from the direct swirl measurements. The ability to 
compute satisfactory values for the mass flow rate, momentum and swirl number from the planar PIV measurements close to the valves shows that global integrated quantities of importance for engineering and industrial purposes can be extracted from PIV and related to specific flow features.

Finally, the stability in time of the swirling structure obtained far from the cylinder head is analysed by tracking the position of the instantaneous swirl centre in each PIV snapshot. The probability density functions obtained with two different cylinder heads show that flow stability is degraded when using a low swirl cylinder head. This could be expected to have consequences on the level of cycle-to-cycle variations for the engine, and thereby affect engine performance.

\section{Acknowledgement}

The present study was carried out in a collaboration between Scania CV AB and KTH. The experiments (Vernet, 2012; Rabault, 2015) were made at Scania CV AB R\&D centre in Södertälje, Sweden and Manuel Schellhase is acknowledged for valuable help during the experiments. Funding and support by Scania is also gratefully acknowledged. Alfredsson was partially funded by the Swedish Foundation for Strategic Research (SSF) under its Strategic Mobility program.

\section{References}

Banaeizadeh, A., Afshari, A., Schock, H., Jaberi, F., 2013. Large-eddy simulations of turbulent flows in internal combustion engines. Int J Heat Mass Tran 60, 781-796.

Basha, S.A., Gopal, K.R., 2009. In-cylinder fluid flow, turbulence and spray models - a review. Renew Sust Energ Rev 13, 1620-1627.

Baum, E., Peterson, B., Böhm, B., Dreizler, A., 2014. On the validation of LES applied to internal combustion engine flows: Part 1: Comprehensive experimental database. Flow Turbulence Combust 92, 269-297.

Baum, E., Peterson, B., Surmann, C., Michaelis, D., Böhm, B., Dreizler, A., 2013. Investigation of the 3D flow field in an IC engine using tomographic PIV. Proc Combust Inst 34, 2903-2910.

Bendat, J.S., Piersol, A.G., 2000. Random Data: Analysis and Measurement Procedures. 3rd ed., John Wiley \& Sons, Inc., New York, NY, USA. Berson, A., Michard, M., Blanc-Benon, P., 2009. Vortex identification and tracking in unsteady flows. C R Mecanique 337, 61-67.

Bizon, K., Continillo, G., Mancaruso, E., Merola, S., Vaglieco, B., 2010. POD-based analysis of combustion images in optically accessible engines. Combust Flame 157, 632-640.

Borée, J., Maurel, S., Bazile, R., 2002. Disruption of a compressed vortex. Phys Fluids 14, 2543-2556.

Bottone, F., Kronenburg, A., Gosman, D., Marquis, A., 2012. Large eddy simulation of diesel engine in-cylinder flow. Flow Turbulence Combust $88,233-253$.

Brücker, C., 1997. 3D scanning PIV applied to an air flow in a motored engine using digital high-speed video. Meas Sci Technol 8, 1480.

Cao, Z.M., Nishino, K., Mizuno, S., Torii, K., 2000. PIV measurement of internal structure of diesel fuel spray. Exp Fluids 29, S211-S219.

Cosadia, I., Borée, J., Charnay, G., Dumont, P., 2006. Cyclic variations of the swirling flow in a diesel transparent engine. Exp Fluids 41 , $115-134$.

Cosadia, I., Borée, J., Dumont, P., 2007. Coupling time-resolved PIV flow-fields and phase-invariant proper orthogonal decomposition for the description of the parameters space in a transparent diesel engine. Exp Fluids 43, 357-370.

Crnojevic, C., Decool, F., Florent, P., 1999. Swirl measurements in a motor cylinder. Exp Fluids 26, 542-548.

Desantes, J., Benajes, J., Urchueguia, J., 1995. Evaluation of the non-steady flow produced by intake ports of direct injection diesel engines. Exp Fluids 19, 51-60.

Dierksheide, U., Meyer, P., Hovestadt, T., Hentschel, W., 2002. Endoscopic 2D particle image velocimetry (PIV) flow field measurements in IC engines. Exp Fluids 33, 794-800.

Enaux, B., Granet, V., Vermorel, O., Lacour, C., Thobois, L., Dugu, V., Poinsot, T., 2011. Large eddy simulation of a motored single-cylinder piston engine: Numerical strategies and validation. Flow Turbulence Combust 86, 153-177.

Enotiadis, A., Vafidis, C., Whitelaw, J., 1990. Interpretation of cyclic flow variations in motored internal combustion engines. Exp Fluids 10, 77-86.

Finn, L.I., Boghosian, B.M., 2006. A global variational approach to vortex core identification. Physica A 362, 11-16.

Fogleman, M., Lumley, J., Rempfer, D., Haworth, D., 2004. Application of the proper orthogonal decomposition to datasets of internal combustion engine flows. J Turbulence $5, \mathrm{~N} 23$.

Garth, C., Laramee, R., Tricoche, X., Schneider, J., Hagen, H., 2007. Extraction and visualization of swirl and tumble motion from engine simulation data, in: Hauser, H., Hagen, H., Theisel, H. (Eds.), Topology-based Methods in Visualization. Springer Berlin Heidelberg. Mathematics and Visualization, pp. 121-135.

Graftieaux, L., Michard, M., Grosjean, N., 2001. Combining PIV, POD and vortex identification algorithms for the study of unsteady turbulent swirling flows. Meas Sci Technol 12, 1422.

Hasse, C., Sohm, V., Durst, B., 2009. Detached eddy simulation of cyclic large scale fluctuations in a simplified engine setup. Int J Heat Fluid Fl $30,32-43$.

Huang, R., Huang, C., Chang, S., Yang, H., Lin, T., Hsu, W., 2005. Topological flow evolutions in cylinder of a motored engine during intake and compression strokes. J Fluid Struct 20, 105-127. 
Huang, R., Yang, H., Yeh, C.N., 2008. In-cylinder flows of a motored four-stroke engine with flat-crown and slightly concave-crown pistons. Exp Therm Fluid Sci 32, 1156-1167.

Kamimoto, T., Kobayashi, H., 1991. Combustion processes in diesel engines. Prog Energ Combust 17, $163-189$.

Kang, K.Y., Reitz, R.D., 1999. The effect of intake valve alignment on swirl generation in a DI diesel engine. Exp Therm Fluid Sci $20,94-103$.

Kapitza, L., Imberdis, O., Bensler, H., Willand, J., Thévenin, D., 2010. An experimental analysis of the turbulent structures generated by the intake port of a DISI-engine. Exp Fluids 48, 265-280.

Khalighi, B., 1991. Study of the intake tumble motion by flow visualization and particle tracking velocimetry. Exp Fluids 10, $230-236$.

Kida, S., Miura, H., 1998. Identification and analysis of vortical structures. Eur J Mech B - Fluid 17, 471-488.

Liu, D., Wang, T., Jia, M., Wang, G., 2012. Cycle-to-cycle variation analysis of in-cylinder flow in a gasoline engine with variable valve lift. Exp Fluids 53, 585-602.

Liu, K., Haworth, D., 2010. Large-eddy simulation for an axisymmetric piston-cylinder assembly with and without swirl. Flow Turbulence Combust 85, 279-307.

Liu, K., Haworth, D., Yang, X., Gopalakrishnan, V., 2013. Large-eddy simulation of motored flow in a two-valve piston engine: POD analysis and cycle-to-cycle variations. Flow Turbulence Combust 91, 373-403.

Lumley, J.L., 2001. Early work on fluid mechanics in the IC engine. Annu Rev Fluid Mech 33, 319-338.

Montorfano, A., Piscaglia, F., Schmitt, M., Wright, Y., Frouzakis, C.E., Tomboulides, A.G., Boulouchos, K., Onorati, A., 2015. Comparison of direct and large eddy simulations of the turbulent flow in a valve/piston assembly. Flow Turbulence Combust , 1-20.

Müller, S., Böhm, B., Gleißner, M., Grzeszik, R., Arndt, S., Dreizler, A., 2010. Flow field measurements in an optically accessible, direct-injection spray-guided internal combustion engine using high-speed PIV. Exp Fluids 48, 281-290.

Murali Krishna, B., Mallikarjuna, J., 2010. Comparative study of in-cylinder tumble flows in an internal combustion engine using different piston shapes - an insight using particle image velocimetry. Exp Fluids 48, 863-874.

van Overbrüggen, T., Dannemann, J., Klaas, M., Schröder, W., 2013. Holographic particle image velocimetry measurements in a four-valve combustion engine. Exp Fluids 55.

Payri, F., Benajes, J., Margot, X., Gil, A., 2004. CFD modeling of the in-cylinder flow in direct-injection diesel engines. Comput Fluids 33, 995-1021.

Payri, F., Desantes, J.M., Pastor, J.V., 1996. LDV measurements of the flow inside the combustion chamber of a 4-valve D.I. diesel engine with axisymmetric piston-bowls. Exp Fluids 22, 118-128.

Peterson, B., Sick, V., 2009. Simultaneous flow field and fuel concentration imaging at $4.8 \mathrm{kHz}$ in an operating engine. Appl Phys B 97 , 887-895.

Pozorski, J., Sazhin, S., Waclawczyk, M., Crua, C., Kennaird, D., Heikal, M., 2002. Spray penetration in a turbulent flow. Flow Turbulence Combust 68, 153-165.

Rabault, J., 2015. PIV Investigation of the Intake Flow in a Parallel Valves Diesel Engine Cylinder. Accessible online: http://www.divaportal.org/smash/get/diva2:843998/FULLTEXT01.pdf. Master's thesis. KTH Mechanics, Stockholm, Sweden.

Reeves, M., Garner, C., Dent, J., Halliwell, N., 1996. Particle image velocimetry analysis of IC engine in-cylinder flows. Opt Laser Eng 25, 415-432.

Reitz, R., Rutland, C., 1995. Development and testing of diesel engine CFD models. Prog Energ Combust 21, $173-196$.

Reitz, R.D., 2013. Directions in internal combustion engine research. Combust Flame 160, 1-8.

Roisman, I., Araneo, L., Tropea, C., 2007. Effect of ambient pressure on penetration of a diesel spray. Int J Multiphas Flow 33, 904-920.

Roudnitzky, S., Druault, P., Guibert, P., 2006. Proper orthogonal decomposition of in-cylinder engine flow into mean component, coherent structures and random Gaussian fluctuations. J Turbulence 7, N70.

Roy, O., Penven, L., 1998. Compression of a turbulent vortex flow. Int J Heat Fluid Fl 19, 533-540.

Schmitt, M., Frouzakis, C.E., Tomboulides, A.G., Wright, Y.M., Boulouchos, K., 2014. Direct numerical simulation of multiple cycles in a valve/piston assembly. Phys Fluids 26, 035105.

Söder, M., 2015. Creation and destruction of in-cylinder flows : Large eddy simulations of the intake and the compression strokes. Ph.D. thesis. KTH Mechanics, Stockholm, Sweden.

Stansfield, P., Wigley, G., Justham, T., Catto, J., Pitcher, G., 2007. PIV analysis of in-cylinder flow structures over a range of realistic engine speeds. Exp Fluids 43, 135-146.

Sun, J., Yates, D., Winterbone, D., 1996. Measurement of the flow field in a diesel engine combustion chamber after combustion by cross-correlation of high-speed photographs. Exp Fluids 20, 335-345.

Toledo, M.S., Penven, L.L., Buffat, M., Cadiou, A., Padilla, J., 2007. Large eddy simulation of the generation and breakdown of a tumbling flow. Int J Heat Fluid Fl 28, 113-126.

Towers, D.P., Towers, C.E., 2004. Cyclic variability measurements of in-cylinder engine flows using high-speed particle image velocimetry. Meas Sci Technol 15, 1917.

Vernet, J., 2012. Detailed study of steady incylinder flow and turbulence using stereo-PIV. Accessible online: http://www.divaportal.org/smash/get/diva2:512553/fulltext01. Master's thesis. KTH Mechanics, Stockholm, Sweden.

Voisine, M., Thomas, L., Borée, J., Rey, P., 2011. Spatio-temporal structure and cycle to cycle variations of an in-cylinder tumbling flow. Exp Fluids 50, 1393-1407.

Waleffe, F., 1990. On the three-dimensional instability of strained vortices. Phys Fluids A 2, 76-80.

Zegers, R., Luijten, C., Dam, N., de Goey, L., 2012. Pre- and post-injection flow characterization in a heavy-duty diesel engine using high-speed PIV. Exp Fluids 53, 731-746. 